\title{
Pacific
}

Journal of

Mathematics

\section{DUALITY FOR FINITE BIPARTITE GRAPHS (WITH AN APPLICATION TO II $_{1}$ FACTORS)}

\section{MARIE CHODA}




\section{DUALITY FOR FINITE BIPARTITE GRAPHS (WITH AN APPLICATION TO II 1 FACTORS)}

\section{MARIE Choda}

Let $\Gamma$ be a finite graph with bicolored vertices and $\theta$ a colorpreserving automorphism of $\Gamma$. We define the dual graph $\widehat{\Gamma}=$ $\widehat{\Gamma}(\theta)$ of $\Gamma$ by $\theta$ and the dual $\hat{\theta}$ of $\theta$ which is an automorphism of $\widehat{\Gamma}$. Under some conditions, $\widehat{\hat{\Gamma}}$ is isomorphic to $\Gamma$. A bicolored graph gives two weighted graphs. The following pair of graphs treated in Index theory are dual pairs: $\left\{\right.$ Coxeter graph of type $A_{2 n-3}$, $\left.D_{n}\right\},\left\{A_{2 n-5}^{(1)}, D_{n}^{(1)}\right\},\left\{E_{6}^{(1)}, E_{7}^{(1)}\right\},\left\{D_{l}^{(1)}, D_{2 l-2}^{(1)}\right\}$, and $\{4$-star $\left.S(1,1, k+1, k+1), \Gamma_{k}\right\}$. The graph of type $D_{4}$ or $E_{6}$ is self dual, but as a weighted graph, the dual of it is another one.

As applications, we have two kinds of outer automorphisms with the period 2 on inclusions of hyperfinite II $_{1}$ factors, one of which gives the inclusion of the crossed products isomorphic to the original one and the other gives the inclusion not isomorphic to the original one.

1. Introduction. Since Jones [7] introduced the index theory for subfactors of finite factors, it is pointed out by several authors that the Dynkin diagrams of Type $A, D, E$ have an important role as an invariant for the classification of the subfactors of the approximately finite dimensional continuous finite factor with index less than 4 (cf. [5], [3], [9] [10], [11], [12], [14]). The relation appears as the diagram for the pair of relative commutant algebras which was defined by Bratteli [2]. He used a finite bipartite graph in order to describe a pair of finite dimensional $C^{*}$-algebras (that is, multi-matrix algebras). Today, such a graph is called a Bratteli diagram. On the other hand, in connection with duality theorems in the theory of locally compact groups, many authors following Takesaki [19] obtained duality theorems for the crossed product of operator algebras by locally compact groups.

From such a viewpoint, in this paper, we shall give a duality theorem for certain bipartite finite graphs. We treat a finite graph $\Gamma$ with bicolored vertices, with an automorphism $\theta$, under which every vertex $v$ has the same color as $\theta(v)$.

Let us consider the graph $\Gamma$ as a Bratteli diagram of the algebras 
$B \subset A$ and $\theta$ an automorphism of $A$ with $\theta(B)=B$. Describing the Bratteli diagram for the crosed products $\langle\theta\rangle \triangleright B \subset\langle\theta\rangle \triangleright A$ as a graph, we shall define the dual graph $\widehat{\Gamma}=\widehat{\Gamma}(\theta)$ of the graph $\Gamma$ by $\theta$ and the dual automorphism $\hat{\theta}$ on $\widehat{\Gamma}$. (We remark that Roche [16] treats a similar graph which is defined by a symmetry of a given graph from the point of view in the conformal field theory.) Under some conditions, we shall show that there exists an isomorphism $\phi$ from $\widehat{\hat{\Gamma}}$ onto $\Gamma$ with the property $\phi \cdot \hat{\hat{\theta}}=\theta \cdot \phi$.

Some kinds of connected bipartite graphs are useful to construct an irreducible pair of $\mathrm{II}_{1}$ factors with small indices. It is necessary to give weights for all vertices of the graph in connection with the trace of the factor. Let mat $\Gamma$ be the matrix defined from a bicolored finite graph $\Gamma$. Using the Perron Frobenius eigenvector of $($ mat $\Gamma)(\text { mat } \Gamma)^{t}$ and $(\text { mat } \Gamma)^{t}($ mat $\Gamma)$, we give a positive number called a weight to each vertex of $\Gamma$. For the dual $\hat{\Gamma}$ of a weighted bicolored graph $\Gamma$ by a color and weight preserving automorphism, we shall define the dual weights for vertices of $\widehat{\Gamma}$ and show the comparability relations between dual weights and eigenvectors with respect to dual graphs. In general, $\widehat{\Gamma}$ is not isomorphic to $\Gamma$, but we have

$$
\| \text { mat } \Gamma\|=\| \text { mat } \widehat{\Gamma} \| \text {. }
$$

It implies that two indices of subfactors coincide if they are given from a dual pair of connected finite bipartite graphs. The graph $\widehat{\hat{\Gamma}}$ is isomorphic to $\Gamma$ as bicolored weighted graphs.

We shall apply those results to graphs appearing in Index theory of finite factors. The first class of them are the Coxeter graphs and the general Coxeter graphs discussed in [3]. The second is the graph by Wenzl ([20]). The third class are two kinds of graphs called $m$-star and $\Gamma_{k}$ by Haagerup and Schou ([4]). We investigate relations in duality among them. In order to have a non-trivial color preserving automorphism, the graph has to be one of the following types: $A_{l}$ $(l \geq 3), A_{l}^{(1)}(l \geq 3), B_{l}^{(1)}, D_{l}(l \geq 4), D_{l}^{(1)}(l \geq 4), E_{6}, E_{6}^{(1)}, E_{7}^{(1)}$, $m$-stars $(m \geq 3), \Gamma_{k}(k \geq 0)$ and Wenzl's graph $\Gamma_{w}$.

Those graphs $\Gamma$ except $B_{l}^{(1)}$ are all isomorphic to $\widehat{\Gamma}$. In the following pair, one is the dual of the other under some symmetry: \{the graph of type $\left.A_{2 n-3}, D_{n}\right\},\left\{A_{2 n-5}^{(1)}, D_{n}^{(1)}\right\},\left\{D_{l}^{(1)}, D_{2 l-2}^{(1)}\right\},\left\{E_{6}^{(1)}, E_{7}^{(1)}\right\}$, \{4-star $S(1,1,1, k), 3$-star $S(k+1, k+1, k+1)\}, \quad\{4$-star $\left.S(1,1, k+1, k+1), \Gamma_{k}\right\}$, and $\left\{5\right.$-star $\left.S(1,1,1,2,2), \Gamma_{w}\right\}$. The graph of type $E_{6}$ is self dual under some automorphisms, but the dual 
of a weighted graph $E_{6}$ is the graph $E_{6}$ with another weights.

In the last section, we shall apply those results to the theory of classification of subfactors. Jones' index was introduced as a conjugacy invariant for subfactors of type $\mathrm{II}_{1}$ factors. Let $N \subset M$ be a pair of type $\mathrm{II}_{1}$ factors and $\theta$ a periodic outer automorphism of $M$ with $\theta(N)=N$. Then the new pair of crossed products $\langle\theta\rangle \triangleright N \subset\langle\theta\rangle \triangleright M$ satisfies $[\langle\theta\rangle \triangleright M:\langle\theta\rangle \triangleright N]=[M: N]$. With respect to the conjugacy problem, it will be natural to investigate whether or not the new pair is isomorphic to the original pair. We give two kinds of examples of outer automorphisms with period 2 for inclusions of hyperfinite type II 1 factors.

First, we shall show that the graph $\Gamma$ is the principal graph of the pair $B(\Gamma) \supset C(\Gamma)$ of hyperfinite $\mathrm{II}_{1}$ factors constructed from the graph $\Gamma$ by the method in $[3, \S 4.4]$, if $\Gamma$ is one of the Coxeter graphs of type $A_{n}, D_{2 n}, E_{6}$ or $E_{8}$. Combining this fact and the duality result of bipartite graphs, we have the following: Let $N \subset M$ have the principal graph $\Gamma$ of type $A_{4 n-3}$ (resp. $D_{2 n}$ ), then the symmetry $\theta$ of $\Gamma$ induces an outer automorphism $\theta$ of $M$ with $\theta(N)=N$ so that the inclusion $\langle\theta\rangle \triangleright M\langle\theta\rangle \triangleright N$ is of type $D_{2 n}$ (resp. $A_{4 n-3}$ ). If $N \subset M$ has the principal graph $D_{4}$ or $E_{6}$, then the symmetry $\theta$ induces that $N \subset M$ so that $\langle\theta\rangle \triangleright N \subset\langle\theta\rangle \triangleright M$ is of type $D_{4}$ or $E_{6}$ respectively.

The author would like express her thanks to Kawahigashi, Kosaki and Ocneanu for valuable discussions, and to Jones and Le Dung Trang for comments and pointing out the references [17] and [18]. She also thanks the referee for reading her manuscript carefully and making appropriate suggestions.

2. Dual graph and dual automorphism. Let $\Gamma$ be a graph, two vertices of which are connected by at most one edge. Let $V(\Gamma)$ be the set of vertices of $\Gamma$ and $E(\Gamma)$ the set of edges. The edge combining $v$ and $w$ in $V(\Gamma)$ is denoted by $(v, w)$. A graph $\Gamma$ with bicolored vertices means that two vertices joined by an edge are colored in such a way that one is black and the other is white. We denote by $B(\Gamma)$ the set of vertices colored by black and by $W(\Gamma)$ the set of vertices with white color. An automorphism $\theta$ of $\Gamma$ is a mapping from $V(\Gamma)$ onto $V(\Gamma)$ which satisfies that if $(v, w) \in E(\Gamma)$ then $(\theta(v), \theta(w)) \in E(\Gamma)$ for all pairs of $v, w \in V(\Gamma)$. When $\theta(v)$ has the same color as $v$ for all $v \in V(\Gamma), \theta$ is said to be color-preserving.

Throughout this paper, $\theta$ is a color-preserving automorphism of a finite graph $\Gamma$ with bicolored vertices. The period $p=p(\theta)$ of $\theta$ is 
the smallest number satisfying that $\theta^{p}$ is the identity on $\Gamma$. For a $v \in V(\Gamma)$, we put

$$
\begin{gathered}
q(v)=\min \left\{k ; \theta^{k}(v)=v\right\}, \quad r(v)=\frac{p}{q(v)}, \\
\hat{v}=\left\{\theta^{k}(v) ; 1 \leq k \leq q(v)\right\} .
\end{gathered}
$$

Let us consider $r(v)$ abstract elements $\hat{v}(0), \hat{v}(1), \ldots, \hat{v}(r(v)-1)$ and identify $\hat{v}(0)$ with $\hat{v}$.

Definition 1. The dual graph $\widehat{\Gamma}=\widehat{\Gamma}(\theta)$ of $\Gamma$ by $\theta$ has the set

$$
V(\widehat{\Gamma})=\{\hat{v}(i) ; v \in \Gamma, 0 \leq i \leq r(v)-1\}
$$

as vertices. Two vertices $\hat{v}(i)$ and $\hat{w}(j)$ in $V(\widehat{\Gamma})$ are combined by an edge in $E(\widehat{\Gamma})$ if there exist $v^{\prime} \in \hat{v}$ and $w^{\prime} \in \hat{w}$ such that $\left(v^{\prime}, w^{\prime}\right) \in$ $E(\Gamma)$, and

$$
\{i q(v)+k ; 0 \leq k \leq q(v)-1\} \cap\{j q(w)+l ; 0 \leq l \leq(w)-1\} \neq \varnothing .
$$

The $\hat{v}(i)$ is given the same color with $v \in V(\Gamma)$ for all $i$.

DEFINITION 2. Let $\left\{\boldsymbol{r}_{k}\right\}_{k}$ be a strictly increasing sequence of natural numbers which satisfies that $r_{i} / r_{i-1}$ is a natural number for all $i$ and that $r_{1}=1$. Then the permutation $\sigma_{k}$ on $\left\{0,1, \ldots, r_{k}-1\right\}$ is defined as follows; for an $i \quad\left(0 \leq i \leq r_{k}-1\right)$, let $\sigma_{k}(2 ; i)=i+$ $r_{k} / r_{2},\left(\bmod r_{k}\right)$. Assume $\sigma_{k}(j ; i)$ is defined for all $j(2 \leq j \leq l-1)$. Let $\sigma_{k}(l ; i)=\sigma_{k}(l-1 ; i)+r_{k} / r_{l},\left(\bmod r_{k} / r_{l-1}\right)$. We define

$$
\sigma_{k}(i)=\left\{\begin{array}{ll}
\sigma_{k}(l ; i), & \text { if } r_{k}-r_{k} / r_{l-1} \leq i<r_{k}-r_{k} / r_{l} \text { for some } l, \\
0, & \text { if } i=r_{k}-1 .
\end{array} .\right.
$$

Condition (A). If $q(v)<q(w)$ for $v, w \in V(\Gamma)$, then there exists a natural number $n$ with $q(w)=n q(v)$.

We need this condition to obtain such an automorphism $\hat{\theta}$ of $\widehat{\Gamma}(\theta)$ that the set $\{\hat{v}(i) ; 0 \leq i \leq r(v)-1\}$ is globally invariant for all $v \in$ $V(\Gamma)$ and that $q(\hat{v}(i))=r(v)$, for all $v \in V(\Gamma)$ and $0 \leq i \leq r(v)-1$. Assume $\theta$ satisfies the Condition (A). We arrange the set $\{q(v) ; v \in$ $V(\Gamma)\}=\left\{p(\theta)=q_{1}>q_{2}>\cdots>q_{n} \geq 1\right\}$, where $q_{i}=q(v)$ for some $v \in V(\Gamma)$. Then $q_{i+1}$ divides $q_{i}$ for all $i$. Let $r_{k}=p(\theta) / q_{k}$, and $\sigma_{k}$ be the permutation of $\left\{0,1, \cdots, r_{k}-1\right\}$ in Definition 2 . 
Definition 3. Assume $\theta$ satisfies the condition (A). The map $\hat{\theta}$ on $\widehat{\Gamma}$ is defined by

$$
\hat{\theta}(\hat{v}(i))=\hat{v}\left(\sigma_{k}(i)\right)
$$

for a $v \in V(\Gamma)$ with $q(v)=q_{k}$ for some $k$.

Proposition 1. The map $\hat{\theta}$ is a color-preserving automorphism of the graph $\widehat{\Gamma}=\widehat{\Gamma}(\theta)$ and $q(\hat{v}(i))=r(v)$ for all $i$. If $\theta$ has a fixed vertex, then $p(\hat{\theta})=p(\theta)$.

Proof. Since $\sigma_{k}$ is the permutation of the set $\left\{0,1, \ldots, r_{k}-1\right\}$ for all $k=1,2, \ldots, n$, it is obvious that $\hat{\theta}$ is a color-preserving one to one map from $V(\widehat{\Gamma})$ onto $V(\widehat{\Gamma})$.

Let $(\hat{v}(i), \hat{w}(j)) \in E(\widehat{\Gamma})$ for some $v$ and $w$ in $V(\Gamma)$. Then we may suppose that $(v, w) \in E(\Gamma)$ and that $\{i q(v)+k ; 0 \leq k \leq q$ $(v)-1\} \cap\{j q(w)+l ; 0 \leq l \leq q(w)-1\} \neq \varnothing$. In the case where $q(v)=q(w)$, we have $i=j$. Let $k$ be $q_{k}=q(v)$. Then

$$
(\hat{\theta}(\hat{v}(i)), \hat{\theta}(\hat{w}(j)))=\left(\hat{v}\left(\sigma_{k}(i)\right), \hat{w}\left(\sigma_{k}(j)\right)\right) \in E(\widehat{\Gamma}) .
$$

In the case where $q(v) \neq q(w)$, we may assume $q(v)>q(w)$. Let $q(v)=q_{k}$ and $q(w)=q_{k+h}$ for some $k(1 \leq k<n)$ and $h(1 \leq$ $h \leq n-k)$. Since $\left\{i q_{k}+m ; 0 \leq m \leq q_{k}-1\right\} \cap\left\{j q_{k+h}+l ; 0 \leq l \leq\right.$ $\left.q_{k+h}-1\right\} \neq \varnothing$, the condition (A) implies that $\left\{i q_{k}+m ; 0 \leq m \leq\right.$ $\left.q_{k}-1\right\} \supset\left\{j q_{k+h}+l ; 0 \leq l \leq q_{k+h}-1\right\}$. Thus $(\hat{v}(i), \hat{w}(j)) \in \bar{E}(\widehat{\Gamma})$ if and only if $j=\left(r_{k+h} / r_{k}\right) i+m$ for some $m\left(0 \leq m \leq\left(r_{k+h} / r_{k}\right)-1\right)$. Assume that $0 \leq i<r_{k}-1$. By Definition $2 r_{k+h}-\left(r_{k+h} / r_{k+l+1}\right)$ for some $l \quad(1 \leq l<h)$, then $\sigma_{k+h}(j)=\left(r_{k+h} / r_{k}\right) \sigma_{k}(i)+m_{l}$. Here, $m_{l}$ is defined by the following method: Let $m_{1}=m+r_{k+h} / r_{k+1}$ $\left(\bmod r_{k+h} / r_{k}\right)$. If $m_{p}$ is defined for all $p(1 \leq p<l)$, let $m_{l}=m_{l-1}+$ $r_{k+h} / r_{k+l}\left(\bmod r_{k+h} / r_{k+l-1}\right)$. Hence, $(\hat{\theta}(v(i)), \hat{\theta}(\hat{w}(j)))=\left(\hat{v}\left(\sigma_{k}(i)\right)\right.$, $\left.\hat{w}\left(\sigma_{k+h}(j)\right)\right) \in E(\widehat{\Gamma})$. Thus, $\hat{\theta}$ is an automorphism of $\widehat{\Gamma}$. It is clear that $r(v)=q(\hat{v}(i))$ for all $i$.

3. Duality for bicolored graphs. In this section, we shall show that, under some conditions, the second dual $\widehat{\hat{\Gamma}}$ is isomorphic to the original graph $\Gamma$. The first condition is the condition (A) for an automorphism $\theta$. The other conditions are the following two conditions $(B)$ and $(C)$.

Condition (B). If there exists such a $k$ that $\left(w, \theta^{k}(v)\right) \in E(\Gamma)$ for $v, w \in V(\Gamma)$ with $q(v)=q(w)$, then $\left(w, \theta^{l}(v)\right) \notin E(\Gamma)$ for all $l \neq k$. 
Condition (C). There are no pairs $\left\{\left(v_{i}, w_{i}\right) \in B(\Gamma) \times W(\Gamma) ; i=\right.$ $1,2\}$ which satisfy the following two conditions:

(a) $q\left(v_{1}\right)=q\left(w_{1}\right)>q\left(v_{2}\right)=q\left(w_{2}\right)>1$.

(b) The sets $\hat{v}_{1}$ and $\hat{w}_{1}$ are joined by edges, $\hat{v}_{1}$ and $\hat{w}_{2}$ are joined by edges, $\hat{w}_{1}$ and $\hat{v}_{2}$ are joined by edges, and $\hat{v}_{2}$ and $\hat{w}_{2}$ are joined by edges.

REMARK. The dual $\widehat{\Gamma}$ always satisfies the Condition (B) for the dual automorphism $\hat{\theta}$. The pairs $(\Gamma, \theta)$ treated in $\S 5$ satisfy the three conditions.

THEOREM 2. Under Conditions (A), (B) and (C), there exists an isomorphism $\phi$ from $\widehat{\hat{\Gamma}}$ onto $\Gamma$ with $\phi \cdot \hat{\hat{\theta}}=\theta \cdot \phi$.

Proof. First, we arrange $V(\Gamma)$ using the following method. Let $\{q(v) ; v \in V(\Gamma)\}=\left\{p=q_{1}>q_{2}>\cdots>q_{n}>0\right\}$. We put $\{\hat{v} ; v \in$ $V(\Gamma), q(v)=p(\theta)\}=\left\{\hat{v}_{1}, \hat{v}_{2}, \ldots, \hat{v}_{m} ; \hat{v}_{i} \neq \hat{v}_{j}(i \neq j)\right\}$. Take an $v(1,0) \in \hat{v}_{1}$ and put $v(1, j)=\theta^{j}(v(1,0))$. Then $\hat{v}_{1}=\{v(1, j) ; 0 \leq$ $j \leq p(\theta)-1\}$. Assume that the set $\hat{v}_{i}$ is put in order for all $0 \leq i<$ $k \leq m$. If there are no edges combining a vertex in $\hat{v}_{k}$ and one in $\hat{v}_{1} \cup \cdots \cup \hat{v}_{k-1}$, then we arrange $\hat{v}_{k}$ by the same method as $\hat{v}_{1}$. In the case where $\hat{v}_{k}$ contains a vertex combined by an edge with some vertex in $\hat{v}_{i}$ for some $i<k$, then by Condition (B), there exists a unique vertex which is adjacent to $v(i, 0)$. We denote the vertex by $v(k, 0)$ and put $v(k, j)=\theta^{j}(v(k, 0))$.

Assume that the set $\left\{v \in V(\Gamma) ; q(v)>q_{k}\right\}$ are arranged in the above order. In the case where there are no vertices $v$ with $q(v)=q_{k}$ and $(v, w) \in E(\Gamma)$ for some $w \in V(\Gamma)$ with $q(w)>q_{k}$, then we arrange the set $\left\{v ; q(v)=q_{k}\right\}$ by the same method as $\{v ; q(v)=$ $p(\theta)\}$.

Assume there exists such a vertex $v$ with $q(v)=q_{k}$ that $(v, w) \in$ $E(\Gamma)$ for some $w$ with $q(w)>q_{k}$. For the $w$, let $\hat{w}=\{w(0)$, $w(1), \ldots, w(q(w)-1)\}$. Take a $v(0) \in \hat{v}$ which is combined by an edge with $w(0)$. Put $\hat{v}=\left\{v(j) ; v(j)=\theta^{j}(v(0))\right.$ for $0 \leq j \leq$ $q(v)-1\}$. Then, by Condition (C), we arrange the set $\left\{v ; q(v)=q_{k}\right\}$ by the same method as for $\{v ; q(v)=p(\theta)\}$.

Now we shall define the map $\phi$. Since, the dual automorphism $\hat{\theta}$ of $\widehat{\Gamma}$ satisfies Condition (A), we can define the dual automorphism $\hat{\hat{\theta}}$ on the graph $\widehat{\hat{\Gamma}}$. For a $z \in V(\widehat{\widehat{\Gamma}})$, let $x \in V(\Gamma)$ be $\hat{z}=\{\hat{x}(0), \hat{x}(1), \ldots, \hat{x}(r(x)-1)\}$. Then $\hat{\theta}(\hat{x}(i))=\hat{x}(\sigma(i))$ for the permutation $\sigma$ on the set $\{0,1, \ldots, r(x)-1\}$ in Definition 2 and 
$x=\hat{w}(j)$ for some $w=w(z) \in V(\Gamma)$ and $j$. Since the cardinal number $\sharp(\hat{x})$ of $\hat{x}$ is $p / r(x)=r(w)=p / q(w)$, we have $q(z)=r(x)=q(w)$. Let the set $\hat{w}=\{w(0), w(1), \ldots, w(q(w)-1)\}$ be arranged in the above order in $V(\Gamma)$. Then we rearrange the set $\hat{z}$ as $\hat{z}=\{z(0), z(1), \cdots, z(r(x)-1)\}$ by the following method. Put $z(0)=\hat{x}(0)$ and $z(i)=(\hat{\hat{\theta}})^{i}(z(0))$ for all $i \quad(0 \leq i \leq r(x)-1)$. Then $z(i)=\hat{x}\left(\sigma^{i}(0)\right)$. We define a map $\phi$ from $V(\widehat{\widehat{\Gamma}})$ to $V(\Gamma)$ by

$$
\phi(z(i))=w(i), \quad \text { for } i(0 \leq i \leq r(x)-1=q(w)-1)
$$

where $w=w(z)$ and $\hat{w}$ is arranged as $\{w(0), w(1), \ldots, w(q(w)-1)\}$ by the above method. Then $\phi$ is a one to one map from $V(\widehat{\Gamma})$ onto $V(\Gamma)$ such that $\phi(\hat{z})=\hat{w}$ for $w=w(z)$.

For two vertices $z(i)$ and $z^{\prime}(j)$ in $\widehat{\hat{\Gamma}}$, let $x$ and $x^{\prime}$ in $V(\widehat{\Gamma})$ correspond to $z(i)$ and $z^{\prime}(j)$ respectively. Put $w=w(z) \in V(\Gamma)$ and $w^{\prime}=w\left(z^{\prime}\right) \in V(\Gamma)$.

Assume that $\left(z(i), z^{\prime}(j)\right) \in E(\widehat{\widehat{\Gamma}})$. Then $\hat{x}$ contains a vertex combined by an edge with some vertex in $\hat{x}^{\prime}$, so that $\hat{w}$ contains a vertex combined by some edge to a vertex in $\hat{w}^{\prime}$. Hence $\left(w(0), w^{\prime}(0)\right) \in$ $E(\Gamma)$.

If $\sharp(\hat{z})=\sharp\left(\hat{z}^{\prime}\right)$, then $i=j$ by the condition $\left(z(i), z^{\prime}(j)\right) \in E(\hat{\hat{\Gamma}})$. Hence $\left(\phi(z(i)), \phi\left(z^{\prime}(j)\right)\right)=\left(w(i), w^{\prime}(i)\right)=\left(\theta(w(0)), \theta^{i}(w(0))\right) \epsilon$ $E(\Gamma)$. In the case $\sharp(\hat{z}) \neq \sharp\left(\hat{z}^{\prime}\right)$, we may assume that $\sharp(\hat{z})<\sharp\left(\hat{z}^{\prime}\right)$. Let $q=q(z)=\sharp(\hat{z})$ and $q^{\prime}=q\left(w^{\prime}\right)=\sharp\left(\hat{z}^{\prime}\right)$. Since

$$
\left((\hat{\hat{\theta}})^{i}(z(0)),(\hat{\hat{\theta}})^{j}\left(z^{\prime}(0)\right)\right)=\left(z(i), z^{\prime}(j)\right) \in E(\widehat{\hat{\Gamma}}),
$$

we have $\left(z^{\prime}(0), z\left(\sigma^{i-j}(0)\right)\right)=\left(z^{\prime}(0),(\hat{\hat{\theta}})^{i-j}(z(0))\right)$ is in $E(\widehat{\hat{\Gamma}})$. By the condition that $\sharp(\hat{z})<\sharp\left(\hat{z}^{\prime}\right)$, the vertex in $\hat{z}$ combined by an edge with $z^{\prime}(0)$ must be $z(0)$ only. Hence $z(0)=z\left(\sigma^{i-j}(0)\right)$ because $\left(z(0), z^{\prime}(0)\right) \in E(\widehat{\widehat{\Gamma}})$ and $\left(z\left(\sigma^{i-j}(0)\right), z^{\prime}(0)\right) \in E(\widehat{\hat{\Gamma}})$. It implies that $\sigma^{i-j}(0)=0(\bmod q)$. Let $m$ be a natural number such that $j-i=$ $m q$. Then $\theta^{j}(w(0))=\theta^{i+m q}(w(0))=\theta^{i}(w(0))$, which implies that

$$
\begin{aligned}
\left(\phi(z(i)), \phi\left(z^{\prime}(j)\right)\right) & =\left(\theta^{i}(w(0)), \theta^{j}\left(w^{\prime}(0)\right)\right) \\
& =\left(\theta^{j}(w(0)), \theta^{j}\left(w^{\prime}(0)\right)\right) \in E(\Gamma) .
\end{aligned}
$$

Thus, for each $z \in V(\widehat{\hat{\Gamma}})$ and $i, \phi \cdot \hat{\hat{\theta}}(z(i))=\phi(z(i+1))=w(i+1)=$ $\theta(w(i))=\theta \cdot \phi(z(i))$. 
An extended version of the results in this section to a finite abelian group will be given by Katayama.

4. Dual of weighted graph. In this section, we shall describe a method to give a weight weight $(v)$ for a $v \in V(\Gamma)$ which is essentially investigated in $([3,11])$. Furthermore, we shall define the dual weights for $V(\widehat{\Gamma})$. Let $B(\Gamma)=\left\{b_{1}, b_{2}, \ldots, b_{m}\right\}$ and $W(\Gamma)=$ $\left\{w_{1}, w_{2}, \ldots, w_{n}\right\}$. We denote by $\Gamma^{t}$ the graph which is the same as $\Gamma$ except that $B\left(\Gamma^{t}\right)=W(\Gamma)$ and that $W\left(\Gamma^{t}\right)=B(\Gamma)$. The $m$-by- $n$ matrix mat $\Gamma=\left[x_{i j}\right]_{i j}$ is defined from $\Gamma$ by

$$
x_{i j}= \begin{cases}1, & \text { if }\left(b_{i}, w_{j}\right) \in E(\Gamma), \\ 0, & \text { otherwise }\end{cases}
$$

We remark that $\theta$ is a color-preserving automorphism of $\Gamma^{t}$ and mat $\widehat{\Gamma}^{t}=(\text { mat } \widehat{\Gamma})^{t}$, where $X^{t}$ is the transposed matrix of a matrix $X$.

Two $m$-by- $n$ matrices $X_{1}$ and $X_{2}$ are said to be pseudo-equivalent if appropriate exchanges of rows and columns convert $X_{1}$ to $X_{2}$.

LEMMA 3. The following two conditions are equivalent:

(1) Two connected graphs $\Gamma_{1}$ and $\Gamma_{2}$ are isomorphic.

(2) $\operatorname{mat}\left(\Gamma_{1}\right)$ is pseudo-equivalent to $\operatorname{mat}\left(\Gamma_{2}\right)$ or $\operatorname{mat}\left(\Gamma_{2}^{t}\right)$.

Definition 4. Let $\Gamma$ be a weighted finite graph with bicolored vertices and $\theta$ a color and weight preserving automorphism of $\Gamma$. Let $x \in V(\widehat{\Gamma})$ be $x=\hat{v}(i)$ for some $v \in V(\Gamma)$ and $i(0 \leq i \leq r(v)-1)$. Then the weight $(x)$ of $x$ is given by

$$
\text { weight }(x)=q(v) \text { weight }(v) \text {. }
$$

The column vectors $s=(s(w))_{w}$ and $t=(t(b))_{b}$ is defined by $s(w)=\operatorname{weight}(w), w \in W(\Gamma), \quad t(b)=\operatorname{weight}(b), \quad b \in B(\Gamma)$, and called the weight vector for $W(\Gamma)$ and $B(\Gamma)$ respectively. We denote the weighted vectors for $W(\widehat{\Gamma})$ and $B(\widehat{\Gamma})$ defined from the weighted vectors $s$ and $t$ as above by $\hat{s}$ and $\hat{t}$.

THEOREM 4. Let $s$ and $t$ be the weight vector for $W(\Gamma)$ and $B(\Gamma)$ respectively. Assume that $\theta$ satisfies Conditions (A), (B) and preserves the weights. If $(\operatorname{mat} \Gamma) s=t$, then $(\operatorname{mat} \widehat{\Gamma}) \hat{s}=\hat{t}$.

Proof. Let $W(\Gamma)=\left\{w_{1}, w_{2}, \ldots, w_{n}\right\}$. For a $b \in B(\Gamma)$ and a $j(0 \leq j \leq r(b)-1)$, put $S(b)=\left\{k ;\left(b, w_{k}\right) \in E(\Gamma)\right\}$ and $S(b, j)=$ 
$\left\{(k, l) ;\left(\hat{b}(j), \hat{w}_{k}(l)\right) \in E(\hat{\Gamma})\right\}$. It is sufficient to prove that, for each $j(0 \leq j \leq r(b)-1)$,

$$
\text { weight }(\hat{b}(j))=\sum_{(k, l) \in S(b, j)} \operatorname{weight}\left(\hat{w}_{k}(l)\right) \text {. }
$$

Since $(\operatorname{mat} \Gamma) s=t$, we have weight $(b)=\sum_{k \in S(b)}$ weight $\left(w_{k}\right)$. Hence

$$
\operatorname{weight}(\hat{b}(j))=\operatorname{weight}(b) q(b)=\sum_{k \in S(b)} q(b) \operatorname{weight}\left(w_{k}\right) .
$$

Put $\{\hat{v} ; v \in W(\Gamma)\}=\left\{\hat{w}_{1}, \hat{w}_{2}, \ldots, \hat{w}_{m} ; \hat{w}_{i} \neq \hat{w}_{j}(i \neq j)\right\}$. Let

$$
S(b) / \theta=\left\{h ;(b, w) \in E(\Gamma) \text { for some } w \in \hat{w}_{h}\right\} .
$$

Assume that $q\left(w_{k}\right) \leq q(b)$ for a $k \in S(b) / \theta$. Then $\hat{w}_{k}$ contains only one vertex combined with $b$ by Conditions (A), (B) and Definition 1. Similarly if $q\left(w_{k}\right)>q(b)$ for a $k \in S(b) / \theta$, the set $\hat{w}_{k}$ contains more vertices than 2 : Let

$$
\begin{aligned}
& S(b)^{\prime}=\left\{k \in S(b) / \theta ; q\left(w_{k}\right) \leq q(b)\right\}, \\
& S(b)^{\prime \prime}=\left\{l \in S(b) / \theta ; q\left(w_{l}\right)>q(b)\right\} .
\end{aligned}
$$

Then

$$
\begin{aligned}
\sum_{k \in S(b)} & q(b) \text { weight }\left(w_{k}\right) \\
= & \sum_{k \in S(b) / \theta} q(b) \text { weight }\left(w_{k}\right) \sharp\left\{w \in \hat{w}_{k} ;(b, w) \in E(\Gamma)\right\} \\
= & \sum_{k \in S(b)^{\prime}} q(b) \operatorname{weight}\left(w_{k}\right) \\
& +\sum_{k \in S(b)^{\prime \prime}} q(b) \cdot \sharp\left\{w \in \hat{w}_{k} ;(b, w) \in E(\Gamma)\right\} \cdot \operatorname{weight}\left(w_{k}\right) \\
= & \sum_{S(b)^{\prime}} \frac{p}{r(b)} \operatorname{weight}\left(w_{k}\right)+\sum_{S(b)^{\prime \prime}} q(b) \cdot \frac{q\left(w_{k}\right)}{q(b)} \cdot \operatorname{weight}\left(w_{k}\right) \\
= & \sum_{S(b)^{\prime}} \frac{r\left(w_{k}\right)}{r(b)} \cdot q\left(w_{k}\right) \cdot \operatorname{weight}\left(w_{k}\right)+\sum_{S(b)^{\prime \prime}} q\left(w_{k}\right) \cdot \operatorname{weight}\left(w_{k}\right) \\
= & \sum_{k \in S(b)} \sharp\left\{l ;\left(\hat{b}(j), \widehat{w_{k}}(l)\right) \in E(\widehat{\Gamma})\right\} \cdot \operatorname{weight}\left(\widehat{w_{k}}(l)\right) \\
= & \sum_{(k, l) \in S(b, j)} \operatorname{weight}\left(\widehat{w_{k}}(l)\right) .
\end{aligned}
$$

Thus the equality $\left({ }^{*}\right)$ holds. It implies that (mat $\left.\widehat{\Gamma}\right) \hat{s}=\hat{t}$.

Let $\Gamma$ be a finite connected graph with labelled bicolored vertices. 
Then there exists a Perron-Frobenius eigen (column) vector $u$ for $($ mat $\Gamma)(\operatorname{mat} \Gamma)^{t}$ by [3]. Let $s=(\operatorname{mat} \Gamma)^{t} u$ and $t=$ mat $\Gamma s$. We give a weight for $b_{i} \in B(\Gamma)$ by

$$
\text { weight }\left(b_{i}\right)=t(i), \quad \text { for } t=(t(i))_{i}
$$

and for $w_{k} \in W(\Gamma)$ by

$$
\text { weight }\left(w_{k}\right)=s(k), \quad \text { for } s=(s(k))_{k} \text {. }
$$

We identify weighted vectors for $W(\Gamma)$ and $B(\Gamma)$ with eigenvectors $s$ and $t$, and denote by the same notation.

Theorem 5. Assume that $\Gamma$ is connected. Let $u$ be a PerronFrobenius eigenvector for (mat $\Gamma)(\text { mat } \Gamma)^{t}$ and $s=(\text { mat } \Gamma)^{t} u$. If $\theta$ preserves the weights of $V(\Gamma)$ defined by $u$ and $s$, then $\hat{u}$ (resp. $\hat{s})$ is a Perron-Frobenius eigenvector for (mat $\widehat{\Gamma})(\text { mat } \widehat{\Gamma})^{t}$ (resp. $(\text { mat } \widehat{\Gamma})^{t}($ mat $\widehat{\Gamma})$ ), under the conditions $(\mathrm{A}),(\mathrm{B})$ and $(\mathrm{C})$. Furthermore,

$$
\| \text { mat } \Gamma\|=\| \text { mat } \widehat{\Gamma} \| \text {. }
$$

Proof. An eigenvalue for $($ mat $\Gamma)(\text { mat } \Gamma)^{t}$ is an eigenvalue for $(\operatorname{mat} \widehat{\Gamma})(\text { mat } \widehat{\Gamma})^{t}$ and $\hat{u}$ (resp. $\left.\hat{s}\right)$ is an eigenvector for $($ mat $\widehat{\Gamma})(\text { mat } \widehat{\Gamma})^{t}$ (resp. (mat $\widehat{\Gamma})^{t}($ mat $\widehat{\Gamma})$ ), which belongs to the original eigenvalue. Since the Perron-Frobenius eigenvalue is the maximum in the set of eigenvalues and the eigenvectors corresponding to it is unique up to the multiple of positive real numbers, it is sufficient to prove that the set of eigenvalues for $($ mat $\Gamma)(\text { mat } \Gamma)^{t}(\operatorname{resp} \text {. (mat } \Gamma)^{t}($ mat $\left.\Gamma)\right)$ is the same as one of $($ mat $\widehat{\Gamma})(\text { mat } \widehat{\Gamma})^{t}\left(\operatorname{resp} .(\operatorname{mat} \widehat{\Gamma})^{t}(\right.$ mat $\left.\widehat{\Gamma})\right)$.

Under the conditions (A), (B) and (C), the graph $\widehat{\hat{\Gamma}}$ is isomorphic to the original one $\Gamma$. Furthermore, the isomorphism in Theorem 2 of $\widehat{\hat{\Gamma}}$ onto $\Gamma$ transposes $B(\widehat{\widehat{\Gamma}})$ and $W(\widehat{\hat{\Gamma}})$ onto $B(\Gamma)$ and $W(\Gamma)$, respectively. It implies that mat $\widehat{\Gamma}$ is pseudo equivalent to mat $\Gamma$ by Lemma 3. Let $m$ and $n$ be the cardinal numbers of $B(\Gamma)$ and $W(\Gamma)$ respectively. Let $\phi$ and $\psi$ be permutations of $\{1,2, \ldots, m\}$ and $\{1,2, \ldots, n\}$ respectively, which implies that mat $\widehat{\hat{\Gamma}}=\phi \cdot$ mat $\Gamma$. $\psi$. Then $($ mat $\widehat{\hat{\Gamma}})(\text { mat } \widehat{\hat{\Gamma}})^{t}=\phi \cdot($ mat $\Gamma)(\text { mat } \Gamma)^{t} \cdot \phi^{t}$. Hence the Perron-Frobenius eigenvalue for $($ mat $\Gamma)(\text { mat } \Gamma)^{t}$ is also that for $($ mat $\widehat{\Gamma})(\text { mat } \widehat{\Gamma})^{t}$, which equals to $\|$ mat $\Gamma\left\|^{2}=\right\|($ mat $\Gamma)(\text { mat } \Gamma)^{t} \|=$ $\|$ mat $\widehat{\Gamma} \|^{2}$. 
COROllary 6. Assume $\Gamma$ is weighted and $\theta$ is weight preserving. Then under conditions (A), (B) and (C), the isomorphism $\phi$ from $\hat{\hat{\Gamma}}$ to $\Gamma$ in Theorem 2 satisfies

$$
\operatorname{weight}(\phi(x))=p(\theta) \operatorname{weight}(x), \quad(x \in V(\widehat{\hat{\Gamma}})) .
$$

Proof. Let $\phi$ be the isomorphism from $\widehat{\hat{\Gamma}}$ onto $\Gamma$ in the proof of Theorem 2. Then $\phi$ preserves the color. Let $x \in V(\widehat{\hat{\Gamma}})$. Then $\phi(x)=v \in V(\Gamma)$ satisfies, for $i(0 \leq i \leq r(v)-1)$ and $j(0 \leq j \leq$ $r(\hat{v}(i))-1)$, that $x=\widehat{\hat{v}(i)}(j)$ where $r(\hat{v}(i))$ is one with respect to $\hat{\theta}$. Since $\operatorname{weight}(\widehat{\hat{v}(i)}(j))=q(\hat{v}(i)) \operatorname{weight}(\hat{v}(i))=r(v) q(v)$ weight $(v)=$ $p(\theta)$ weight $(v), \phi$ satisfies the relation.

REMARK. Corollary 6 means that $\widehat{\hat{\Gamma}}$ is essentially the original graph $\Gamma$ as weighted graphs, because the Perron-Frobenius eigenvector is unique up to scalar multiples.

5. The graphs in the index theory. In this section, we shall restrict our interest to the graph $\Gamma$ which appeared in the recent results of the index theory for type $\mathrm{II}_{1}$-factors. A typical class of them are Coxeter graphs and general Coxeter graphs. The second class is the graph treated by Haagerup and Schou [4] to give a new irreducible pair of $\mathrm{II}_{1}$ factors with small indices. They called those graphs $\mathrm{m}$ stars $(m \geq 2)$. Coxeter graphs appear as a special kind of $m$-star for $m=2$, or 3. They ([4]) announced the graph which gives an irreducible pair of factors with index $r(4<r<5)$ by their method is either one of 4-stars or of $\Gamma_{k}(k \geq 1)$. Another interesting graph is the diagram obtained by Wenzl [20], which we denote by $\Gamma_{w}$.

For the graphs of the following type, the color preserving automorphism is only the identity : $B_{l}(l \geq 2), E_{7}, E_{8}, F_{4}, G_{2}, H_{i}(i=$ $3,4), I_{2}(p), A_{1}^{(1)}, C_{l}^{(1)}(l \geq 2), F_{5}, G_{2}^{(1)}, E_{8}^{(1)}$

We give the weights for $V(\Gamma)$ by the method in $\S 4$. Then we have the dual pairs as the following table (see next page). In the table, for the not self dual pair, $\widehat{\Gamma}$ is unique as a weighted graph. So we do not denote the weights for such pairs. In the table we put $\lambda=$ $\left(4 \cos ^{2} \pi / 12\right)^{-1}$ and $\mu=1 / 3$. The $m$-star $S\left(k_{1}, \ldots, k_{m}\right)$ in the table has $m$ "rays" from the central point and the number of vertices on the $i$ th ray (not counting the central point) is $k_{i}(1 \leq i \leq m)$. The graph $\Gamma_{k}$ has $k+4$ vertices. Then all automorphisms $\theta$ and $\hat{\theta}$ in the table preserve the colors, satisfy the conditions (A), (B), (C), and preserve the weights given by the method in $\S 4$. 
TABLE

\begin{tabular}{|c|c|}
\hline$\{r, \theta\} \cong\{\hat{\Gamma}, \widehat{\hat{\theta}}\}$ & $\{\hat{\Gamma}, \hat{\theta}\}$ \\
\hline${ }^{A_{2 n-3}} \leq$ & $D_{n}$ \\
\hline$\lambda \lambda(1-\lambda) \lambda \lambda$ & 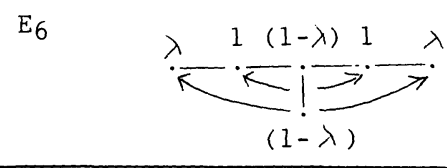 \\
\hline $\mathrm{D}_{4}$ & $\mathrm{D}_{4}$ \\
\hline$A 2 n-5$ & $D_{n}^{(1)}$ \\
\hline$D_{2 n-2}^{(1)}$ & $\mathrm{D}_{\mathrm{n}}^{(1)}$ \\
\hline$E_{\zeta}^{(1)}$ & $E f^{(1)}$ \\
\hline$s(1,1,1, k)$ & $\mathrm{S}(\mathrm{k}+1, \mathrm{k}+1, \mathrm{k}+1$ \\
\hline$\Gamma_{k}$ & $\mathrm{~s}(1,1, \mathrm{k}+1, \mathrm{k}+1)$ \\
\hline$\Gamma_{\mathrm{w}}$ & $\mathrm{S}(1,1,1,2,2)$ \\
\hline
\end{tabular}

6. Application to subfactors. In this section, we shall show some applications of the above results to the principal graphs for subfactors of the hyperfinite type $\mathrm{II}_{1}$ factor. For notions and terminologies for the theory of subfactors, see for example [3, Chapters 3 and 4]. Two pairs $N_{i} \subset M_{i}(i=1,2)$ are said to be isomorphic if there exists a 
*-isomorphism $\phi$ of $M_{1}$ onto $M_{2}$ with $\phi\left(N_{1}\right)=N_{2}$. Let us consider the pair of tensor products with the same matrix algebra. Then it is isomorphic to the original pair, because the two towers of the relative commutants are isomorphic. In this section we shall investigate the pair of crossed products. As an application of the duality of graphs, we shall give two kinds of examples of crossed products by an outer automorphism with the period 2, one of which gives a pair isomorphic to the original one, and another of which gives a pair non isomorphic to the original one. First, we have the following fact, which is clear by the relation between the conditional expectations of the original pair and the crossed product.

LEMMA 7. Let $N \subset M$ be a pair of type $\mathrm{II}_{1}$ factors and $G$ a finite group of outer automorphisms of $M$ with $g(N)=N$ for all $g \in G$. Then $[G \triangleright M: G \triangleright N]=[M: N]$.

Let $N \subset M$ be a pair of type $\mathrm{II}_{1}$ factors with index $[M: N]<\infty$ and finite depth. Then the principal graph for $N \subset M$ is defined in [3] as the Bratteli diagram for the inclusion of the tower of relative commutant algebras of $N$ in the tower of basic constructions. It is a bipartite graph with the weights on the vertices, which are given by the restriction of the Markov trace of the basic extension algebras. Two pairs of hyperfinite $\mathrm{II}_{1}$ factors are isomorphic if and only if the canonical commuting squares are isomorphic ([14]). If $[M: N]<4$, then the principal graph is one of the Coxeter graphs of type $A_{n}, D_{n}, E_{6}, E_{7}, E_{8}$ ([3], [11], [14]), and $D_{\text {odd }}, E_{7}$ do not appear as principal graphs ([5], [9], [11], [12]).

Let $\Gamma$ be one of the Coxeter graphs $A_{n}, D_{n}, E_{6}, E_{7}$ or $E_{8}$. A pair of hyperfinite $\mathrm{II}_{1}$ factors is given from $\Gamma$ by the method of $[3, \S 4.4]$ as follows:

Let $C_{0} \subset B_{0}$ be a pair of finite dimensional von Neumann algebras with $\Gamma$ as the Bratteli diagram of them and the minimal central projections of $C_{0}$ correspond with $W(\Gamma)$. Here, we have two choices of $W(\Gamma)$ for each $\Gamma$. As $\Gamma$ is connected, there is a unique normalized Markov trace $\tau$ for the pair $C_{0} \subset B_{0}$. The trace vectors of $\tau$ on $C_{0}$ and $B_{0}$ are given by a Perron-Frobenius eigenvector of $(\operatorname{mat} \Gamma)(\text { mat } \Gamma)^{t}$ by the method in $\S 4$. Let $e_{0}$ be the projection of $L^{2}\left(B_{0}, \tau\right)$ onto $L^{2}\left(C_{0}, \tau\right)$ and $B_{1}=\left\langle B_{0}, e_{0}\right\rangle$, the basic extension for the inclusion $C_{0} \subset B_{0}$. Let $B_{i}=\left\langle B_{i-1}, e_{i-1}\right\rangle$ be the basic extension of $B_{i-2} \subset B_{i-1}$ for $i \geq 2$ and $C_{i}$ the von Neumann subalgebra in $B_{i}$ generated by $C_{i-1}$ and $e_{i-1}$. Let us take a $q$ with $q+q^{-1}+2=\|\operatorname{mat}(\Gamma)\|^{2}$. Then a unitary $w_{i} \in B_{i+1}$ is defined by 
$(q+1) e_{i}-1$. The trace $\tau$ is extended to $\bigcup_{i=0}^{+\infty} B_{i}$ and gives a hyperfinite II $_{1}$ factor $B(\Gamma)$ by the GNS construction. Similarly the sequence $\left(C_{i}\right)_{i \geq 0}$ gives a subfactor $C(\Gamma)$ of $B(\Gamma)$. Then we have the following theorem.

TheOREM 8. Let $\Gamma$ be one of the Coxeter graphs $A_{n}, D_{2 m}, E_{6}$ or $E_{8}$. Then the principal graph of $B(\Gamma) \supset C(\Gamma)$ is $\Gamma$.

Proof. The inclusion matrices of $B_{j} \subset B_{j+1}$ are alternately (mat $\left.\Gamma\right)^{t}$ and mat $\Gamma$. The inner automorphism $\phi_{n}=\operatorname{Ad} w_{0} w_{1} \cdots w_{n}$ of $B_{n+1}$ preserves $C_{n}$ globally invariant and $\phi_{n}\left(B_{n}\right)=C_{n+1}$. Through $\phi_{n}$ the Bratteli diagrams of $C_{n} \subset C_{n+1}$ and $C_{n+1} \subset B_{n+1}$ are determined. Let the distinguished point $*$ be a vertex in $\Gamma$ with the smallest entry of the Perron-Frobenius eigenvector. Let $p$ be a one dimensional projection in the subalgebra of some $C_{k}$, which has the minimal central projection corresponding to the distinguished point *. Remark that $k$ is chosen as 0 or 1 . Let $N$ be the reduction algebra $C(\Gamma)_{p}$ and $M=B(\Gamma)_{p}$. Then the pair $C(\Gamma) \subset B(\Gamma)$ is conjugate to $N \subset M$ by [22]. On the other hand, the unitaries $w_{0}$ and $w_{1}$ give a complex number to each cell which corresponds to the pair $\left(q_{0} r_{0} r_{1}, q_{0} r_{0}^{\prime} r_{1}\right)$ and $\left(q_{1} r_{1} r_{2}, q_{1} r_{1}^{\prime} r_{2}\right)$, where $q_{0} \in C_{0}$ and $r_{i}, r_{i}^{\prime} \in B_{i}(i=0,1,2)$ are minimal central projections respectively. These numbers satisfy the biunitarity axiom of connections due to Ocneanu, because they are unitaries induced by Jones' projections $e_{0}$ and $e_{1}$. The renormalization rule comes from the definition of $e_{0}$ and $e_{1}$ for the pair $C_{0} \subset B_{0}$ and $B_{0} \subset B_{1}$ respectively. Thus for each $n,\left(B_{n}\right)_{p}$ is considered as the algebra given by $(n+1)$-strings starting from the distinguished point * and $\left(C_{n}\right)_{p}$ as the subslgebra given by the embedding of the $n$-strings algebra starting from $*$ by $\operatorname{Ad}\left(w_{n-1}\right)_{p}\left(w_{n-2}\right)_{p} \cdots\left(w_{1}\right)_{p}\left(w_{0}\right)_{p}$. The above connection $W$ must be equivalent to one of those given in [9] and [12]. Since $p$ is contained in $C_{0}$, we have $C(\Gamma)_{p}=\left(\bigcup_{n}\left(C_{n}\right)_{p}\right)^{\prime \prime}$ and $B(\Gamma)_{p}=\left(\bigcup_{n}\left(B_{n}\right)_{p}\right)^{\prime \prime}$. Hence the pair $N=C(\Gamma)_{p} \subset B(\Gamma)_{p}=M$ is considered as the pair of factors obtained by the strings starting from * by Ocneanu's method. On the other hand the connections on $A_{n}, D_{2 n}, E_{6}$ and $E_{8}$ are flat ([6], [9], [12]). Hence the principal graph of the inclusion $N \subset M$ is $\Gamma$ by [12] (cf. [1]). Thus $C(\Gamma) \subset B(\Gamma)$ has $\Gamma$ as the principal graph, because $C(\Gamma) \subset B(\Gamma)$ is conjugate to $N \subset M$.

Lemma 9. Let $\Gamma$ be one of the Coxeter graphs $A_{2 n+1}, D_{n}$ or $E_{6}$. Then the symmetry $\theta$ of $\Gamma$ in the table induces an outer automorphism $\theta$ of $B(\Gamma)$ such that $\theta\left(C_{j}\right)=C_{j}$ and $\theta\left(B_{j}\right)=B_{j}$ for all $j$. 
Proof. It is clear that $\theta$ induces an automorphism $\theta$ of $B(\Gamma)$ such that $\theta\left(B_{j}\right)=B_{j}$ and $\theta\left(e_{j}\right)=e_{j}$ for each Jones projection from $B_{j-1}$ onto $B_{j-2}$ which is defined through the Bratteli diagram. Let $\phi$ be the *-endomorphism defined by

$$
\phi(x)=\lim _{k \rightarrow \infty} \operatorname{Ad} w_{0} w_{1} \cdots w_{k}(x)
$$

for $x \in B(\Gamma)$. Then $\phi \theta=\theta \phi$. Since $C_{j}=\phi\left(B_{j-1}\right)$, we have $\theta\left(C_{j}\right)=C_{j}$, for all $j$. Hence $\theta(C)=C$. By the proof of the next theorem, we show that $\langle\theta\rangle \triangleright B(\Gamma)$ is isomorphic to $B(\widehat{\Gamma}, \theta)$, which is a $\mathrm{II}_{1}$ factor. Hence $\theta$ must be outer.

THEOREM 10. Let $B(\Gamma) \supset C(\Gamma)$ be the pair obtained from $\Gamma$ and $\theta$ the automorphism of $B(\Gamma) \supset C(\Gamma)$ induced by the symmetry of $\Gamma$.

(1) If $\Gamma$ is $A_{4 n-3}$ (resp. $D_{2 n}$ ), then the principal graph of the crossed product $\mathbf{Z}_{2} \triangleright_{\theta} B(\Gamma) \supset \mathbf{Z}_{2} \triangleright_{\theta} C(\Gamma)$ is $D_{2 n}$ (resp. $\left.A_{4 n-3}\right)$.

(2) If $\Gamma$ is $E_{6}$, then $B(\Gamma) \supset C(\Gamma)$ is conjugate to $\mathbf{Z}_{2} \triangleright_{\theta} B(\Gamma) \supset$ $\mathbf{Z}_{2} \triangleright_{\theta} C(\Gamma)$ and the principal graph is $E_{6}$.

Proof. Put $B=B(\Gamma)$ and $C=C(\Gamma)$. Then the pair is determined by the commuting square consisting of $C_{0}, B_{0}, B_{1}, C_{1}$, which are defined above. Let $M$ be the crossed product $\mathbf{Z}_{2} \triangleright_{\theta} B, M_{j}=\mathbf{Z}_{2} \triangleright_{\theta} B_{j}$, $N_{j}=\mathbf{Z}_{2} \triangleright_{\theta} C_{j}$ and $N=\mathbf{Z}_{2} \triangleright_{\theta} C$. Then $N$ (resp. $M$ ) is generated by $\left(N_{j}\right)_{j}$ (resp. $\left.\left(M_{j}\right)_{j}\right)$. We have the unitary $u$ in $N_{0}$ with $\theta(x)=u x u^{*}$ for all $x$ in $B$. Then $N_{j}^{\prime} \cap N_{j}=\left\{a+b u: a \in\left(C_{j}^{\prime} \cap C_{j}\right)\right.$ with $\theta(a)=$ $a, b=\sum_{p} b_{p}$ for minimal central projections $p \in C_{j}$ with $\left.\theta(p)=p\right\}$. The center of $M_{j}$ is given similarly. Hence by the consequence of the previous section, the Bratteli diagram of the inclusion $N_{0} \subset M_{0}$ is the dual graph $\widehat{\Gamma}=\widehat{\Gamma}(\theta)$. The *-endomorphism $\phi$ of $B$ in Lemma 9 is extended to $M$ and $M_{j}$ for all $j$ by $\phi(x+u y)=\phi(x)+u \phi(y)$ for all $x, y \in B$, because $\theta(\phi(x))=\phi(\theta(x))$ for all $x \in B$. The extension of $\phi_{1}$ to $M_{1}$ is an automorphism of $M_{1}$ with the properties $\phi\left(M_{0}\right)=N_{1}$ and $\phi\left(N_{0}\right)=N_{0}$. Hence the commuting square consisting of $\left\{N_{0}, M_{0}, M_{1}, N_{1}\right\}$ is isomorphic to the commuting square consisting of $\left\{C_{0}(\widehat{\Gamma}), B_{0}(\widehat{\Gamma}), B_{1}(\widehat{\Gamma}), C_{1}(\widehat{\Gamma})\right\}$. Therefore we have $N \subset$ $M$ is conjugate to $C(\widehat{\Gamma}) \subset B(\widehat{\Gamma})$. Then $W(\widehat{\Gamma})$ is defined by Definition 1.

If $\Gamma$ is of type $A_{4 n-3}$ (resp. $D_{2 n}$ ), then the principal graph of $B \supset C$ is $A_{4 n-3}$ (resp. $D_{2 n}$ ), and the principal graph of $M \supset N$ is $D_{2 n}$ (resp. $A_{4 n-3}$ ) by Theoerm 8 and the results in the previous 
section. Hence $\theta$ gives the pair of another type that is $D_{2 n}$ (resp. $\left.A_{2 n-3}\right)$.

Let $\Gamma$ be of type $E_{6}$. Then $\widehat{\Gamma}=E_{6}$. If the distinguished point $*$ is contained in $W(\Gamma)$, then $C_{0}(\widehat{\Gamma})$ corresponds $W(\widehat{\Gamma})$, which is the set of odd vertices of $E_{6}$. The inclusion $N \subset M$ is also determined by the commuting square consisting of $\left\{C_{1}(\widehat{\Gamma}), B_{1}(\widehat{\Gamma}), B_{2}(\widehat{\Gamma}), C_{2}(\widehat{\Gamma})\right\}$, which defines a connection of $\widehat{\Gamma}$ by the method in the proof of Theorem 8. The connection of $E_{6}$ is equivalent to the original connection which gives the pair $B \supset C$. Hence by Theorem 8 and the dual property of $\left(E_{6}, \theta\right)$, the statement $(2)$ is proved by a similar method as (1).

By the relation between the crossed products and fixed point algebras, we have the following as an immediate consequence of Theorem 10.

COROLLARY 11. Let $M \supset N$ be a pair of hyperfinite $\mathrm{II}_{1}$ factors, the principal graph $\Gamma$ of $M \supset N$ be one of the Coxeter graphs $A_{4 n-3}, D_{2 n}$, $E_{6}$ and $\theta$ the symmetry of $\Gamma$.

(1) If $\Gamma$ is $A_{4 n-3}$ (resp. $D_{2 n}$ ), then the principal graph of the pair $M^{\theta} \supset N^{\theta}$ of the fixed point algebras is $D_{2 n}$ (resp. $A_{4 n-3}$ ).

(2) If $\Gamma$ is $E_{6}$, then the principal graph of $N^{\theta} \subset M^{\theta}$ is $E_{6}$.

\section{REFERENCES}

[1] J. Bion-Nadal, Subfactor of the hyperfinite $\mathrm{II}_{1}$ factor with Coxeter graph $E_{6}$ as invariant, Current Topics in Operator algebras, World Scientific, (1991).

[2] O. Bratteli, Inductive limits of finite dimensional $C^{*}$-algebras, Trans. Amer. Math. Soc., 171 (1972), 195-234.

[3] F. M. Goodman, P. Harpe, and V. F. R. Jones, Coxeter Graphs and Towers of Algebras, Springer Verlag, 1989.

[4] U. Haagerup and K. Schou, Some new subfactors of the hyperfinite $\mathrm{II}_{1}$-factor, preprint.

[5] M. Izumi, Application of fusion rules to classification of subfactors, to appear in Publ. RIMS.

[6] _ Flatness of $E_{8}$, Talk at the conference of Yamanakako (1991).

[7] V. F. R. Jones, Index for subfactors, Invent. Math., 72 (1983), 1-25.

[8] _ Braid groups, Hecke algebras and Type $\mathrm{II}_{1}$ factors, Geometric Methods in Operator Algebras, Pitman, Res. Notes in Math., 123 (1986), 242-273.

[9] Y. Kawahigashi, On flatness of Ocneanu's connections on the Dynkin diagrams and classification of subfactors, preprint.

[10] , An analogue of a solvable lattice model in classification of subfactors, preprint. 
[11] A. Ocneanu, Quantized groups, String Algebras and Galois Theory for Algebras, Operator Algebras and Applications, vol. II, London Math. Soc. Lecture Note Series, Cambridge Univ. Press, 136 (1988), 119-172.

[12] __, Quantum symmetry, differential geometry of finite graphs and classification of subfactors, notes by Kawahigashi.

[13] S. Okamoto, Invariants for subfactors arising from Coxeter graphs, preprint.

[14] S. Popa, Classification of subfactors: the reduction to commuting squares, Invent. Math., 101 (1990), 19-43.

[15] __ Sur la classification des sousfacteurs d'indice fini du facteur hyparfini, C.R. Acad. Sci. Paris, 311 (1990), 95-100.

[16] Ph. Roche, Ocneanu cell calculus and integrable lattice model, Comm. Math. Phys., 127 (1990), 395-424.

[17] K. Saito, Extended affine root systems I, Publ. RIMS, Kyoto Univ., 21 (1985), 75-179.

[18] S. Slodowy, Sur les Groupes Finis Attaches aux Singularites Simples, Introduction a la Théorie des Singularités II, Hermann, (1990), 109-125.

[19] M. Takesaki, Duality for crossed products and structure of von Neumann algebras of type III, Acta Math., 131 (1973), 249-310.

[20] H. Wenzl, Quantum groups and subfactors of type B, C, and D, Comm. Math. Phys., 133 (1990), 383-432.

[21] _ Hecke algebras of type $A_{n}$ and subfactors, Invent. Math., 92 (1988), 349383.

[22] S. Popa, Relative dimension, towers of projections and commuting squares of subfactors, Pacific J. Math., 137 (1989), 181-207.

Received October 20, 1990 and in revised form March 15, 1992.

Osaka KyoIKu University

TENNOJI Osaka 543, JAPAN 



\title{
PACIFIC JOURNAL OF MATHEMATICS
}

Founded by

\author{
E. F. BeCKeNBACH (1906-1982) F. Wolf (1904-1989)
}

\section{EDITORS}

\section{S. VARADARAJAN (Managing Editor) University of California Los Angeles, CA 90024-1555 vsv@math.ucla.edu \\ F. Michael Christ University of California Los Angeles, CA 90024-1555 christ@math.ucla.edu}

\section{Herbert Clemens}

University of Utah

Salt Lake City, UT 84112

clemens@math.utah.edu

\author{
THOMAS ENRIGHT \\ University of California, San Diego \\ La Jolla, CA 92093 \\ tenright@ucsd.edu \\ Nicholas ERcolani \\ University of Arizona \\ Tucson, AZ 85721 \\ ercolani@math.arizona.edu \\ R. FINN \\ Stanford University \\ Stanford, CA 94305 \\ finn@gauss.stanford.edu \\ VAUGHAN F. R. JONES \\ University of California \\ Berkeley, CA 94720 \\ vfr@math.berkeley.edu
}

\section{SUPPORTING INSTITUTIONS}

UNIVERSITY OF ARIZONA

UNIVERSITY OF BRITISH COLUMBIA

UNIVERSITY OF OREGON

CALIFORNIA INSTITUTE OF TECHNOLOGY

UNIVERSITY OF CALIFORNIA

UNIVERSITY OF MONTANA

UNIVERSITY OF NEVADA, RENO

NEW MEXICO STATE UNIVERSITY

OREGON STATE UNIVERSITY
SteVen KerckhofF

Stanford University

Stanford, CA 94305

spk@gauss.stanford.edu

MARTIN SCHARLEMANN

University of California

Santa Barbara, CA 93106

mgscharl@henri.ucsb.edu

HAROLD STARK

University of California, San Diego

La Jolla, CA 92093

\author{
STANFORD UNIVERSITY \\ UNIVERSITY OF HAWAII \\ UNIVERSITY OF UTAH \\ WASHINGTON STATE UNIVERSITY \\ UNIVERSITY OF WASHINGTON
}

UIV 


\section{PACIFIC JOURNAL OF MATHEMATICS}

Volume $158 \quad$ No. $1 \quad$ March 1993

Determinant identities

GEORGE W. Eyre ANDrEWS and WiLliam H. Burge

A spectral theory for solvable Lie algebras of operators

E. BOASSO and ANGEL RAFAEL LAROTONDA

Simple group actions on hyperbolic Riemann surfaces of least area

S. Allen Broughton

Duality for finite bipartite graphs (with an application to $\mathrm{II}_{1}$ factors)

MARIE CHODA

Szegő maps and highest weight representations

MARK GREGORY DAVIDSON and Ron STANKE

Optimal approximation class for multivariate Bernstein operators

ZEEV DitZIAN and XINLONG ZHOU

Witt rings under odd degree extensions

ROBERT FITZGERALD

Congruence properties of functions related to the partition function

ANTHONY D. FORBES

Bilinear operators on $L^{\infty}(G)$ of locally compact groups

Colin C. GRAham and Anthony To-Ming LAU

Nonuniqueness of the metric in Lorentzian manifolds

GEOFFREY K. MARTIN and GERARD THOMPSON

Index theory and Toeplitz algebras on one-parameter subgroups of Lie 189 groups

EFTON PARK 